08

\title{
Эффективная интенсивность излучения лазерных импульсов, определяющая вероятность многофотонных процессов
}

\author{
(c) Б.А. Зон, А.С. Корнев \\ Воронежский государственный университет, \\ 394018 Воронеж, Россия \\ ฯ e-mail: a-kornev@yandex.ru \\ Поступила в редакцию 29.04.2020 г. \\ В окончательной редакции 29.04.2020 г. \\ Принята к публикации 04.05.2020 г.
}

\begin{abstract}
Количественно рассмотрено влияние неопределенности интенсивности излучения отдельных лазерных импульсов на вероятности многофотонной ионизации/фотоотрыва. Рассмотрены как многофотонный режим (на примере аниона $\mathrm{F}^{-}$), так и туннельный режим (на примере атома водорода). Показано, что в многофотонном режиме флуктуации интенсивности заметно изменяют вероятности процессов. Что же касается туннельного режима, то роль флуктуаций интенсивности здесь пренебрежимо мала, причем не только в области насыщения, но и вдали от нее.
\end{abstract}

Ключевые слова: многофотонная ионизация, туннельная ионизация, нелинейная статистическая оптика.

DOI: 10.21883/OS.2020.09.49871.143-20

\section{Введение}

Многофотонная ионизация атомов и молекул в сильном лазерном поле активно изучается теоретически и экспериментально уже более полувека, начиная с пионерной теоретической работы Келдыша [1] и первого экспериментального наблюдения эффекта Вороновым и Делоне [2] в атомах благородных газов. Изложению современного состояния проблемы посвящен специальный выпуск журнала [3]. Весьма полезные сведения имеются в работе [4]. Укажем также недавние публикации [5,6], в которых рассматриваются ионизация и возбуждение квантовых систем сверхкороткими импульсами.

На первом этапе экспериментальных исследований использовались одиночные лазерные импульсы, содержащие, как правило, большое число продольных световых мод. По этой причине лазерное излучение существенно отличалось от идеализированных монохроматических световых импульсов. Теоретическому и экспериментальному исследованию влияния многомодовости излучения на вероятность ионизации посвящено значительное число публикаций (например, $[7,8])$. Обзор этих исследований приведен в работе [9] (см. также [10]).

Основным проявлением многомодовости излучения в многофотонном режиме при достаточно большом числе мод в импульсе явилось увеличение вероятности ионизации в $n$ ! раз, где величина $n$ определяет многофотонность процесса. Для туннельного режима ионизации учет многомодовости изменял функциональную зависимость вероятности ионизации от интенсивности излучения. Столь сильное влияние многомодовости в обоих случаях имеет простое физическое объяснение. Оно связано с тем, что при сильной нелинейности процесса его вероятность определяется не средним значением ин- тенсивности, а ее статистическими выбросами, которые достаточно велики для излучения с большим числом мод.

Заметим, что многомодовость излучения важна не только для ионизации, но и для других нелинейных процессов, таких как генерация гармоник [10], деполяризация лазерного излучения в нелинейной среде [11-13], многоквантовый вынужденный тормозной эффект при рассеянии электрона на атоме в присутствии лазерного излучения [14-17]. В последнем случае учет именно многомодовости излучения позволил объяснить экспериментальные результаты, полученные в работе [18].

В настоящее время экспериментальное изучение многофотонных процессов, включая ионизацию атомов и молекул [19], проводятся при использовании последовательности коротких лазерных импульсов, каждый из которых можно с хорошей точностью считать одномодовым. Однако энергии отдельных импульсов могут несколько различаться. Кроме того, источником флуктуаций интенсивности могут являться нестабильности, возникающие при фокусировке излучения в область взаимодействия со средой [20].

В качестве типичного примера подобных экспериментов можно указать работы [21,22], в которых изучался 5- и 6-фотонный фотоотрыв электрона от аниона $\mathrm{F}^{-}$, в результате которого образовывался нейтральный атом $\mathrm{F}$ в основном $P_{3 / 2^{-}}$или возбужденном $P_{1 / 2}$-состоянии соответственно. Для согласования экспериментальных данных с теорией [23] в работе [22] было предположено, что интенсивность излучения, создаваемая лазерными импульсами в области фотоотрыва, превышает среднюю интенсивность до 60\%. При этом погрешность определения средней интенсивности оценивалась в $15 \%$. В качестве одной из основных причин такого расхож- 
дения в работе [10] указывались именно флуктуации интенсивности в области фокусировки излучения. Одной из целей настоящей работы является количественная оценка роли этих флуктуаций.

Помимо многофотонного режима фотоотрыва, изучавшегося в работах [21,22], в данной работе рассматривается также туннельный режим ионизации/фотоотрыва, который в настоящее время чаще исследуется экспериментально. Например, для аниона $\mathrm{F}^{-}$туннельный фотоотрыв экспериментально изучался в работе [24]. Соответствующая теория была предложена в работе [25].

В следующем разделе содержатся аналитические и численные результаты, связанные с проявлениями флуктуаций интенсивности лазерного излучения описанного выше типа в процессах с фиксированной нелинейностью. Аналогичные результаты для туннельного режима изложены в разд. 2. Совместное рассмотрение этих двух режимов в одной работе вполне естественно, поскольку еще в работе [1] было показано, что многофотонный и туннельный режимы ионизации являются предельными случаями одного процесса. В Заключении приведены краткие выводы.

\section{1. Нелинейный процесс $n$-го порядка}

Вероятность $n$-фотонного процесса определяется корреляционной функцией амплитуд электрического поля излучения F, медленно зависящих от времени [26]:

$$
\begin{aligned}
& \mathrm{F}_{F}\left(t_{1}, \ldots, t_{n} ; t_{1}^{\prime}, \ldots, t_{n}^{\prime}\right)= \\
& \left.\quad=\left\langle F\left(t_{1}\right) \ldots F\left(t_{n}\right) F^{*}\left(t^{\prime}\right)_{1}\right) \ldots F^{*}\left(t_{n}^{\prime}\right)\right\rangle
\end{aligned}
$$

Здесь $F^{*}$ означает комплексно сопряженную $F$ величину, угловые скобки соответствуют усреднению по реализациям поля при заданной его интенсивности. Рассмотрим простейший случай линейно поляризованного излучения, когда $F=F^{*}$. Кроме того, на основании сказанного во Введении будем рассматривать полностью когерентное излучение, что позволяет опустить угловые скобки в (1).

Пусть максимум интенсивности $I(t)$, которая пропорциональна $F^{2}(t)$, достигается в момент времени $t=0$. В этом случае функция (1) достигает максимума при $t_{1}=t_{2}=\ldots=t_{n}^{\prime}=0$ [27], и с точностью до постоянного множителя

$$
\mathrm{F}_{F}(0, \ldots, 0) \propto \mathrm{F}_{I}(0, \ldots, 0)=I^{n}(0) .
$$

Будем считать, что интенсивность излучения удовлетворяет распределению Гаусса:

$$
P(I)=\frac{1}{\sqrt{2 \pi} \sigma} \exp \left\{-\frac{1}{2}\left(\frac{I-I_{1}}{\sigma}\right)^{2}\right\} .
$$

Здесь $\sigma^{2}$ - дисперсия; для краткости обозначено $I(0)=I$; параметр

$$
I_{1}=\int_{-\infty}^{+\infty} I P(I) d I
$$

является средней интенсивностью, измеряемой линейным детектором, например калориметром. Предполагается, как обычно, что $I_{1} \gg \sigma$, так что область $I<0$, не имеющая физического смысла, не влияет существенно на значение интеграла (4).

Для нелинейного, порядка $n$, детектора определим соответствующую среднюю величину:

$$
\begin{aligned}
I_{n} & =\left(\int_{-\infty}^{+\infty} I^{n} P(I) d I\right)^{1 / n} \\
& =\sqrt{2} \sigma\left(\frac{1}{\sqrt{\pi}} \int_{-\infty}^{+\infty} x^{n} e^{-\left(x-x_{1}\right)^{2}} d x\right)^{1 / n} \\
& =\frac{\sigma}{\sqrt{2}}\left[\mathrm{i}^{-n} H_{n}\left(\frac{\mathrm{i} I_{1}}{\sqrt{2} \sigma}\right)\right]^{1 / n}, x_{1}=\frac{I_{1}}{\sqrt{2} \sigma} .
\end{aligned}
$$

Здесь $H_{n}$ - полином Эрмита [28]. Как будет показано ниже, $I_{n}$ является эффективной интенсивностью $n$ фотонного процесса, учитывающей флуктуации излучения. Отношение $I_{n}$ к средней интенсивности $I_{1}$ определяет роль флуктуаций. Заметим также, что поскольку выражение в формуле (5) содержит точку ветвления (степень $1 / n)$, в ней нельзя делать кажущиеся очевидными упрощения. При таких ,упрощениях“ можно попасть на лист римановой поверхности, на котором, например, выражение (5) становится комплексным. Вычисления в формуле (5) следует проводить именно в той последовательности, которая в ней определена. Эти вычисления легко проводятся с помощью широко распространенных вычислительных средств, например пакета Mathematica [29].

Зависимость $I_{n} / I_{1}$ от $n$ показана точками на рис. 1 для $\sigma_{0}=0.05,0.15,0.25$, где $\sigma_{0}=\sigma / I_{1}$. При проведении вычислений мы пренебрегли уменьшением плотности анионов в области фокусировки излучения. Иными словами, вычисления проведены вдали от области насыщения. Сплошные кривые на рис. 1 показывают приближение этой зависимости квадратичной функцией

$$
I_{n} / I_{1} \approx a+b n+c n^{2} .
$$

Значения коэффициентов $a, b$ и $c$ приведены в таблице.

Максимальным значением $n$, для которого проводились вычисления, является значение $n=25$, так как, согласно работе [21], при фотоотрыве электрона наблюдается поглощение до 23 фотонов. Как видно, отношение $I_{n} / I_{1}$ возрастает с ростом $n$. Однако даже для наибольшего значения $n$ величина $I_{n}$ отличается 


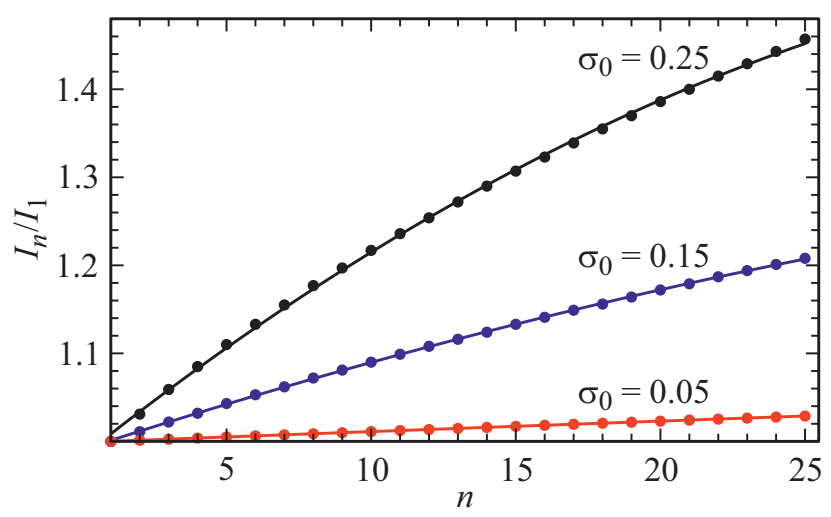

Рис. 1. Зависимости $I_{n} / I_{1}$ от $n$ (точки) для трех указанных значений квадратного корня из дисперсии $\sigma_{0}=\sigma / I_{1}$. Сплошные кривые показывают аппроксимацию этих зависимостей функцией (6).

Значения коэффициентов $a, b$ и $c$ из аппроксимационной формулы (6) для трех значений $\sigma_{0}=\sigma / I_{1}$

\begin{tabular}{c|c|c|c}
\hline$\sigma_{0}$ & 0.05 & 0.015 & 0.025 \\
\hline$a$ & 0.999 & 0.990 & 0.983 \\
$b$ & 0.00125 & 0.0108 & 0.0262 \\
$c$ & $-2.06 \cdot 10^{-6}$ & $-8.38 \cdot 10^{-4}$ & $-2.97 \cdot 10^{-3}$
\end{tabular}

от $I_{1}$ примерно на $20 \%$ для $\sigma_{0}=0.15$, что существенно меньше $60 \%$, необходимых в соответствии с [22] для согласия теории и эксперимента. Следовательно, предположение о том, что флуктуации интенсивности могут быть ответственны за расхождение теории и эксперимента при многофотонном отрыве электрона от аниона $\mathrm{F}^{-}$, представляется не вполне обоснованным. Это, разумеется, не ставит под сомнение успешное сравнение экспериментальных результатов с теорией [23], поскольку помимо неопределенности энергии и интенсивности лазерных импульсов существуют и другие источники экспериментальных погрешностей. Часть из них связана, например, с пространственно-временной метрикой лазерного поля, поскольку нейтральные атомы, образуемые в результате многофотонного отрыва, извлекаются из конечной пространственно-временной области.

\section{2. Туннельная ионизация}

Рассмотрим теперь туннельную ионизацию, при которой происходит переход атома или иона из начального состояния $i$ в конечное состояние $f$. Вероятность такого процесса в единицу времени, усредненная по оптическому периоду, при линейной поляризации излучения определяется соотношением (в атомных единицах)

$$
\begin{aligned}
W_{f i}(F)= & \frac{Q_{f i}^{2} C_{v l}^{2} \kappa^{2}}{2^{|m|}|m !|} \frac{(l+1 / 2)(l+|m|) !}{(l-|m|) !} \\
& \times \sqrt{\frac{3 F}{\pi \kappa^{3}}}\left(\frac{2 \kappa^{3}}{F}\right)^{2 v-|m|-1} \exp \left(-\frac{2 \kappa^{3}}{3 F}\right) .
\end{aligned}
$$

Здесь $F$, как и ранее, является амплитудой электрического поля линейно поляризованного излучения, $l$ и $m-$ соответственно орбитальный момент и магнитное квантовое число туннелирующего электрона,

$$
\kappa=\left[2\left(I+\Delta E_{f}\right)\right]^{1 / 2}
$$

- волновое число туннелирующего электрона, $\mathscr{I}$ потенциал ионизации начального состояния $i, \Delta E_{f}-$ энергия возбуждения рождающегося иона/тома, $Q_{f i}-$ интеграл перекрытия между волновыми функциями связанных электронов в начальном и конечном состояниях. Для $p$-электронов угловые части факторов $Q_{f i}$ приведены в Приложении к работе [30]. Так как факторы $Q_{f i}$ определяются также радиальными частями электронных функций, они позволяют выйти за рамки приближения замороженного остова (концепция орбиталей Дайсона). Неравенства $\Delta E_{f} \neq 0$ и $Q_{f i} \neq 1$ отличают формулу (7) от стандартной модели АДК.

Эффективное главное квантовое число $v$ связано с волновым числом $\kappa$ соотношением $v=Z / \kappa$, где $Z$ - заряд остаточного атома/иона. Вероятность ионизации/отрыва в единицу времени (7) определяется также постоянной $C_{v l}$ в асимптотическом $(r \rightarrow \infty)$ виде волновой функции туннелирующего электрона:

$$
\Psi_{\nu l m}(\mathbf{r})=C_{\nu l} \kappa^{3 / 2}(\kappa r)^{\nu-1} e^{-\kappa r} Y_{l m}(\hat{\mathbf{r}}), \hat{\mathbf{r}}=\mathbf{r} / r .
$$

Учтем теперь, что в атомных единицах (а.и.) $F(t)=\left[I(t) / I_{\mathrm{au}}\right]^{1 / 2}$, где $I_{\text {au }} \cong 3.51 \cdot 10^{16} \mathrm{~W} / \mathrm{cm}^{2}$. Предположим, что зависимость интенсивности от времени $I(t)$ дается функцией Гаусса:

$$
I(t)=I^{(0)} e^{-t^{2} / \tau^{2}},
$$

где параметр $\tau$ определяет длительность лазерного импульса.

Будем считать, что распределение максимальной интенсивности $I(0)$ описывается той же формулой (3), что и в многофотонном случае. Среднее число актов фотоионизации/фотоотрыва за время воздействия лазерного импульса определяется теперь следующим выражением:

$N_{\sigma}(t) / N_{0}=1-\exp \left[-\int_{-\infty}^{t} d t^{\prime} \int_{0}^{\infty} d I_{\text {tun }}^{(0)} P\left(I_{\text {tun }}^{(0)}\right) W_{f i}\left(F\left(t^{\prime}\right)\right)\right]$,

где $N_{0}-$ число атомов или отрицательных ионов в начальном состоянии.

Проведенные вычисления, результаты которых приводятся ниже, показывают, что флуктуации интенсивности 


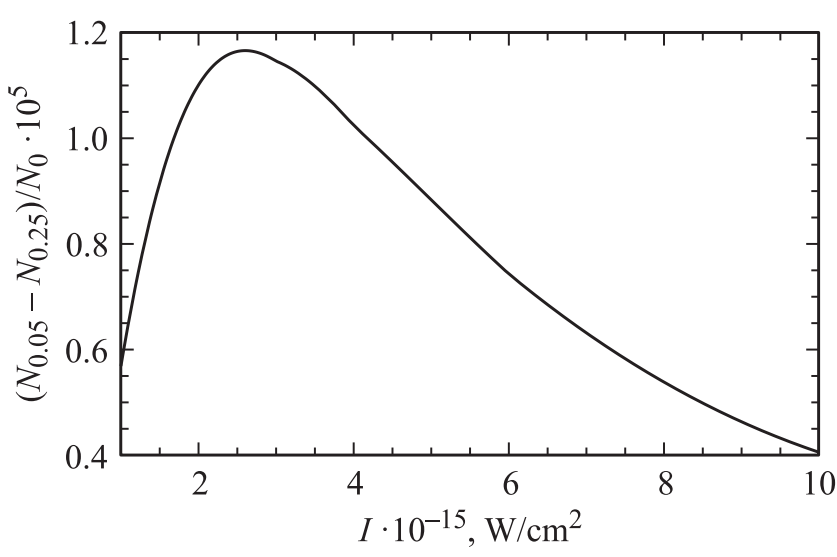

Рис. 2. Относительная разность числа ионов $\mathrm{H}^{+}$, рожденных в результате туннельной ионизации, для $\sigma_{0}=0.05$ и $\sigma_{0}=0.25$. Среднее число ионов вычислялось по формуле (11) при $t \gg \tau$.

лазерных импульсов в туннельном режиме ионизации весьма слабо проявляются в выходе ионов в отличие от многофотонного режима. Это означает, что эффективная интенсивность, рассмотренная в предыдущем разделе для многофотонного режима, в туннельном режиме фактически совпадает со средней интенсивностью, определяющей вероятности линейных процессов.

В качестве примера на рис. 2 представлены результаты вычислений для относительной разности среднего числа ионов $\mathrm{H}^{+},\left(N_{0.05}-N_{0.25}\right) / N_{0}$, для двух значений $\sigma_{0}$ при $t \gg \tau$ при туннельной ионизации атома водорода. В этом случае $l=m=0, v=\kappa=1, Y=0.5$ a.u., $C_{v l}=2$. Для наименьшей из представленных на рисунке интенсивностей, $10^{15} \mathrm{~W} / \mathrm{cm}^{2}$, вероятность ионизации примерно равна $20 \%$, а для наибольшей интенсивности, $10^{16} \mathrm{~W} / \mathrm{cm}^{2}$, вероятность близка к единице. Как видно, величина $\left(N_{0.05}-N_{0.25}\right) / N_{0}$ во всем диапазоне интенсивностей изменяется на уровне $10^{-5}$. Тот факт, что в области насыщения вероятность ионизации не зависит от флуктуаций интенсивности, физически совершенно понятен: в этой области вероятность ионизации слабо зависит от малых изменений интенсивности. Однако практически полное отсутствие такой зависимости вдали от насыщения является несколько неожиданной.

\section{Заключение}

Полученные в работе результаты показывают, что в многофотонном режиме вероятность ионизации/отрыва определяется эффективной интенсивностью, зависящей от величины флуктуаций, и отличающейся от средней интенсивности, определяющей вероятности линейных процессов. Что касается туннельного режима, то вероятность ионизации/отрыва в этом случае практически не зависит от флуктуаций интенсивности, причем во всем диапазоне интенсивностей, а не только в области насыщения.
Физическая причина столь сильного различия роли флуктуаций, рассмотренных в данной работе и в работах [7-17], связана, как говорилось, с важной ролью статистических выбросов интенсивности. Формально это проявляется в том, что в случае многомодового излучения распределение интенсивностей близко к экспоненциальному [7-17], тогда как распределение интенсивности одномодовых импульсов, рассмотренных в настоящей работе, предполагается гауссовым.

\section{Благодарности}

Авторы благодарят И.Ю. Кияна (I.Yu. Kiyan) и Д. Штрассера (D. Strasser) за полезные обсуждения.

\section{Финансирование работы}

Работа выполнена при финансовой поддержке Российского научного фонда (грант № 19-12-00095; расчёты многофотонного режима ионизации) и Российского фонда фундаментальных исследований (грант 19-52-26006; расчёты туннельного режима ионизации).

\section{Конфликт интересов}

Авторы заявляют, что у них нет конфликта интересов.

\section{Список литературы}

[1] Келдыш Л.В. // ЖЭТФ. 1964. Т. 47. № 5. С. 1945; Keldysh L.V. // J. Exp. Theor. Phys. 1965. V. 20. N 5. P. 1307.

[2] Воронов Г.С., Делоне Н.Б. // ЖЭТФ. 1966. Т. 50. № 1. С. 78; Voronov G.S., Delone N.B. // J. Exp. Theor. Phys. 1966. V. 23. N 1. P. 54.

[3] J. Phys. B: At. Mol. Opt. Phys. 2014. V. 47. N 20. Special issue on 50 years of optical tunneling.

[4] Lai Y.H., Xu J., Szafruga U.B., Talbert B.K., Gong X., Zhang K., Fuest H., Kling M.F., Blaga C.I., Agostini P., DiMauro L.F. // Phys. Rev. A. 2017. V. 96. N 6. P. 063417. doi 10.1103/PhysRevA.96.063417

[5] Розанов Н.Н. // Опт. и спектр. 2018. Т. 124. № 1. C. 75. doi 10.21883/OS.2018.01.45361.174-17; Rosanov N.N. // Opt. Spectrosc. 2018. V. 124. N 1. P. 72. doi 10.1134/S0030400X18010186

[6] Розанов Н.Н., Архипов М.В., Архипов Р.М., Веретенов Н.А., Пахомов А.В., Федоров С.В. // Опт. и спектр. 2019. T. 127. № 1. C. 82.

doi 10.21883/OS.2019.07.47934.80-19; Rosanov N.N., Arkhipov M.V., Arkhipov R.M., Veretenov N.A., Pakhomov A.V., Fedorov S.V. // Opt. Spectrosc. 2019. V. 127. N 1. P. 77. doi 10.1134/S0030400X19070221

[7] Карапетян Р.В. // Изв. вузов. Сер. Радиофизика. 1975. T. 18. № 2. C. 236.

[8] Масалов А.В. // Квант. электрон. 1976. Т. 3. № 8. С. 1667; Masalov A.V. // Sov. J. Quantum Electron. 1976. V. 6. N 8. P. 902. doi 10.1070/QE1976v006n08ABEH011743 
[9] Делоне Н.Б., Коварский В.А., Масалов А.В., Перельман Н.Ф. // УФН. 1980. Т. 131. № 8. С. 617. doi 10.3367/UFNr.0131.198008c.0617; Delone N.B., Kovarskii V.A., Masalov A.V., Perel'man N.F. // Sov. Phys.-Usp. 1980. V. 23. N 8. P. 472. doi 10.1070/PU1980v023n08ABEH005021

[10] Ахманов С.А., Дьяков Ю.Е., Чиркин А.С. Введение в статистическую радиофизику и оптику. М.: Наука, 1981. $640 \mathrm{c}$.

[11] Зон Б.А., Кузнецова Н.А. // Опт. и спектр. 1990. Т. 69. № 2. C. 192.

[12] Chernov V.E., Zon B.A. // J. Opt. Soc. Am. B. 1993. V. 10. N 2. P. 210. doi 10.1364/JOSAB. 10.000210

[13] Chernov V.E., Zon B.A. // J. Comput. Meth. Sci. Eng. 2010. V. 10. N 3-6. P. 183. doi 10.3233/JCM-2010-0348

[14] Zoller P.J. // Phys. B. 1980. V. 13. N 8. P. L249. doi 10.1088/0022-3700/13/8/001

[15] Daniele R., Ferrante G.J. // Phys. B. 1981. V. 14. N 20. P. L635. doi 10.1088/0022-3700/14/20/004

[16] Бейлин Е.Л., Зон Б.А. // Квант. электрон. 1982. T. 9. № 8. C. 1692; Beilin E.L, Zon B.A. // Sov. J. Quantum Electron. 1982. V. 12. N 8. P. 1083. doi 10.1070/QE1982v012n08ABEH005795

[17] Daniele R., Faisal F.H.M., Ferrante G. // J. Phys. B. 1983. V. 16. N 20. P. 3831. doi 10.1088/0022-3700/16/20/020

[18] Weingartshofer A., Holmes J.K., Caudle G., Clarke E.M., Krüger H. // Phys. Rev. Lett. 1977. V. 39. N 5. P. 269. doi 10.1103/PhysRevLett.39.269

[19] Дьячков А.Б., Горкунов А.А., Лабозин А.В., Миронов С.М., Панченко В.Я., Фирсов В.А., Цветков Г.О. // Опт. и спектр. 2020. T. 128. № 3. C. 301. doi 10.21883/OS.2020.03.49055.277-19

[20] Киян И.Ю. // Частное сообщение; Kiуan I.Үи. // Private communication.

[21] Kiyan I.Yu., Helm H. // Phys. Rev. Lett. 2003. V. 90. N 18. P. 183001. doi 10.1103/PhysRevLett.90.183001

[22] Bergues B., Ni Y., Helm H., Kiyan I.Yu. // Phys. Rev. Lett. 2005. V. 95. N 26. P. 263002. doi 10.1103/PhysRevLett.95.263002

[23] Gribakin G.F., Kuchiev M.Yu. // Phys. Rev. A. 1997. V. 55. N 5. P. 3760. doi 10.1103/PhysRevA.55.3760

[24] Albeck Y., Lerner G., Kandhasamy D.M., Chandrasekaran V., Strasser D. // Phys. Rev. A. 2015. V. 92. N 6. P. 061401(R). doi 10.1103/PhysRevA.92.061401

[25] Kornev A.S., Zon B.A. // Laser Phys. Lett. 2019. V. 16. N 10. P. 105301. doi 10.1088/1612-202x/ab3d3f

[26] Рапопорт Л.П., Зон Б.А., Манаков Н.Л. Теория многофотонных процессов в атомах. М.: Атомиздат, 1978. 184 с.

[27] Mandel L., Wolf E. Optical Coherence and Quantum Optics. Cambridge University Press, 1995. 1166 p. doi 10.1017/СВО9781139644105; Перевод: Мандель Л., Воль $\oint$ Э. Оптическая когерентность и квантовая оптика. М.: Физматлит, 2000. 896 с.

[28] Bateman H., Erdélyi A. Higher Transcendential Functions. Bateman Manuscript Project. Californian Institute of Technology. V. II. New York-Toronto-London: McGraw-Hill, 1953. 292 р.; Бейтмен Г., Эрдейи А. Высшие трансцендентные функции. Функции Бесселя, функции параболического цилиндра, ортогональные многочлены. М.: Наука, 1966. $296 \mathrm{c}$.

[29] Wolfram S. The Mathematica Book. NY. Wolfram Media, 2003. $1488 \mathrm{p}$.

[30] Kornev A.S., Tulenko E.B., Zon B.A. // Phys. Rev. A. 2003. V. 68. N 4. P. 043414. doi 10.1103/PhysRevA.68.043414 